\title{
Field Quality Measurements of a 2-Tesla Transmission Line Magnet
}

\author{
G. V. Velev, W. Foster, V. Kashikhin, P. Mazur, A. Oleck, H. Piekarz, P. Schlabach, C. Sylvester,
} M. Wake

\begin{abstract}
A prototype 2-Tesla superconducting transmission line magnet for future hadron colliders was designed, built and tested at Fermilab. The $1.5 \mathrm{~m}$ long, combined-function gradientdipole magnet has a vertical pole aperture of $20 \mathrm{~mm}$. To measure the magnetic field quality in such a small magnet aperture, a specialized rotating coil of $15.2 \mathrm{~mm}$ diameter, $0.69 \mathrm{~m}$ long was fabricated. Using this probe, a program of magnetic field quality measurements was successfully performed. Results of the measurements are presented and discussed.
\end{abstract}

Index Terms- Accelerator magnets, Magnetic field measurements, Superconducting magnets

\section{INTRODUCTION}

A $\mathrm{S}$ a main component of every future collider, the magnet system has a strong influence on the total cost of the machine. Because of this, a major goal of machine design is optimization of the cost per Tesla-meter of the collider. A collaboration of institutions of which Fermilab was a member has performed a design study for a staged Very Large Hadron Collider (VLHC) [1]. The first stage of this collider would be built with a 2-in-1, warm-iron, combinedfunction, gradient-dipole magnet excited by a superconducting transmission line.

A cross-section of the magnet is shown in Fig. 1. The laminated core is created from upper and lower half cores stamped from $1 \mathrm{~mm}$ thick low carbon steel. The upper and lower laminations are stacked and welded longitudinally into a yoke. The precise spacing of the two yoke structure is set by non-magnetic stainless steel bars, and the halves are welded together with non-magnetic plates at the inner radius. Details and history of the design are reported elsewhere [2]-[5], the last of which is presented at this conference.

A prototype model of the magnet was fabricated at Fermilab where a new facility was built specifically for testing it [6]. In recent tests the magnet was excited with DC currents up to $105 \mathrm{kA}$ in liquid helium at $4.5 \mathrm{~K}$ [5].

In this paper, we present the results of field harmonics

Manuscript received September 19, 2005. This work was supported by the U.S. Department of Energy.

W. Foster, V. Kashikhin, P. Mazur, A. Oleck, H. Piekarz, P. Schlabach, C. Sylvester and G. V. Velev, are with Fermilab, Batavia, IL 60510 USA (phone: 630-840-3000; fax: 630-840-2383 e-mail: velev@fnal.gov).

M. Wake is with KEK, Tsukuba, 305-0801, Japan.

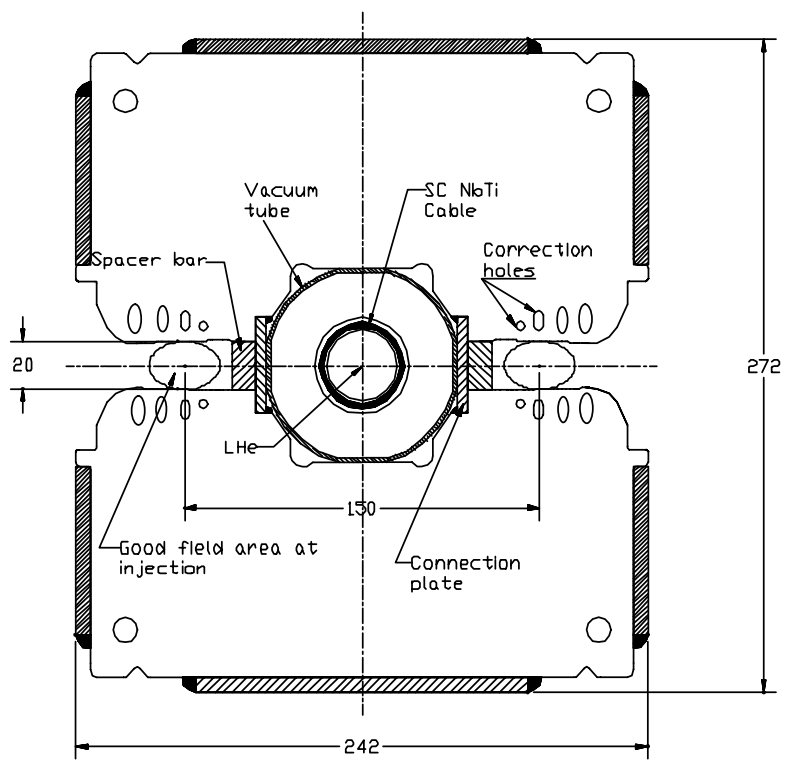

Fig. 1. A cross-section of the transmission line magnet. Dimensions are given in $\mathrm{mm}$.

measurements of the prototype magnet using a rotating coil. Details of the fabrication of the coil built for these measurements are discussed as well. A companion paper, also presented at this conference [7], summarizes the field measurement results obtained from an array of 102 Hall effect sensors.

\section{MEASUREMENT SYSTEM}

Field measurements were performed using a portable magnetic measurement "cart" developed for production testing of LHC insertion region quadrupoles in Fermilab's Magnet Test Facility (MTF). This cart contains DAQ and motor control hardware. The rotating coil, support and positioning fixtures, and drive system were developed for measurement of the prototype magnet.

\section{A. DAQ system}

The DAQ system is based on Metrolab 5035 precision digital integrators (PDI) [8]. Five of these devices read the voltages generated by flux changes in the coil windings. An HP3458 digital volt-meter (DVM) monitors the magnet current. A special trigger module, developed and built inhouse, conditions signals from an angular encoder in the probe drive system and triggers PDIs and DVM, synchronizing simultaneous measurements of field and 


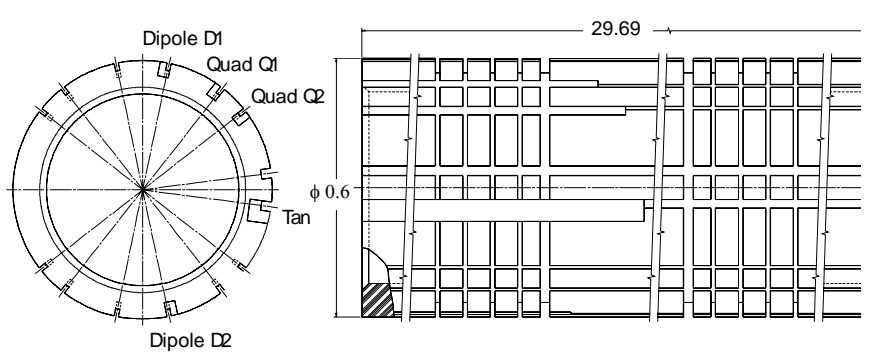

Fig. 2. Cross-section and longitudinal view of the measurement probe. Note the small probe diameter (0.6 in., $15.2 \mathrm{~mm})$.

current. The PDIs are first configured to read and store data in the internal buffers. When the buffers are full, the PDI data are transferred to the PPC 2301 VME computer. During this time, no data are acquired. The live time fraction is $45 \%$. We attempted continuous data streaming from PDIs to PPC but were unable to establish reliable running during long measurements. From the PPC, data are streamed to on-line analysis code running on a PC workstation [9]. The Java code responsible for analysis, data archival, visualization, and measurement control is based on the EMS framework [10]. Details of the readout system are reported elsewhere [11][12].

\section{B. Probe drive system}

The probe is rotated by a stepper motor precisely controlled using feedback from an angular encoder attached to the probe drive shaft. The motor and the encoders are placed as far as possible from the magnet to prevent disruption of motor rotation by the magnet fringe field. The motor is also shielded on 3 sides with 1/8 in. steel. An 8:1 reduction-ratio gearbox is included in the probe drive assembly to smooth the angular steps from the motor. Rotation is transferred from the gearbox to the probe shaft by a serpentine belt. A set of slip rings is connected in line with the probe to extract signals.

\section{Measurement probe}

The measurement probe is of a typical style used in MTF. A tangential winding is sensitive to harmonics of all orders. Two dipole and two quadrupole windings for measurement of the lowest order components of the field are included (Fig. 2). The coil sensitivities were selected such that the large dipole and quadrupole signals from the tangential winding are bucked when combined with those of the dipole and quadrupole windings. The probe diameter is small $(15.2 \mathrm{~mm})$ relative to other probes used at MTF. Typical winding angles would have left minimal material between some winding slots so the standard design was changed to increase this material while maintaining sensitivity factors producing full bucking of the tangential winding signal.

Given the small probe radius, the tangential winding opening angle was optimized to increase signal strength of low order harmonics at the expense of sensitivity to a broad range of orders. For this probe configuration we estimated that a measurement accuracy of 0.1 units through harmonic order

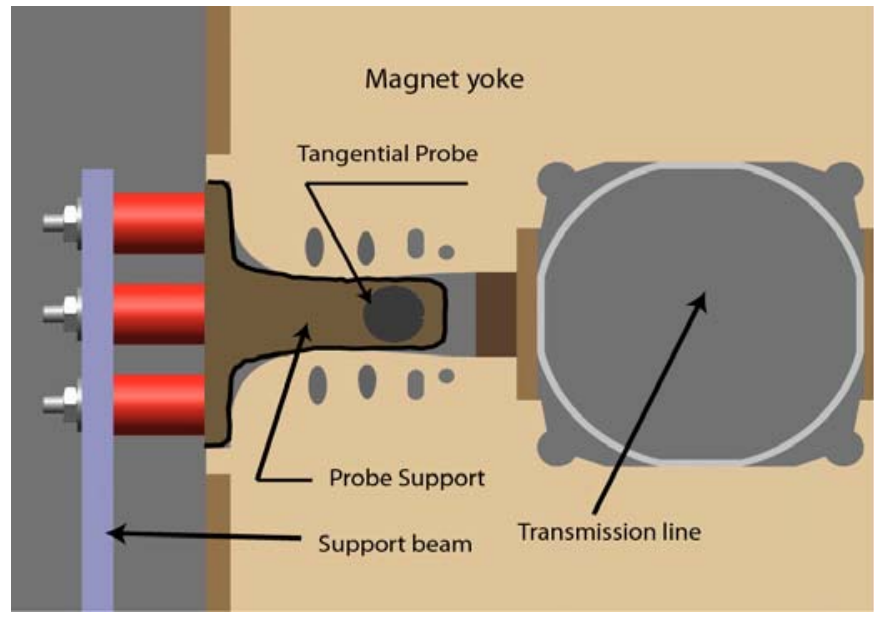

Fig. 3. Half of the cross-section of the transmission line magnet. The arrows show the position of the tangential probe and its support.

10 is achievable at fields greater than $0.3 \mathrm{~T}$.

The length of the probe form is $754 \mathrm{~mm}$ with an active length of $\sim 690 \mathrm{~mm}$. The large aspect ratio makes the structure very compliant requiring support along the length. Vespel ${ }^{\circledR}$ [13] was used to produce the probe form. This polyimide material was selected because of its dimensional stability. It is widely used in bearing applications, performing well without needing lubrication. These characteristics enable us to support and rotate the probe without separate bearing structures.

\section{Probe support assembly}

Due to the small $20 \mathrm{~mm}$ bore of this transmission line magnet, we adopt a new approach for the probe support assembly. The gap size, coupled with the usual desire to maximize the probe radius for best sensitivity, makes it impractical to employ standard techniques and components. In this assembly, there is very little space for such things as a commercial non-magnetic bearing, bearing housing, or provisions for centering the probe assembly.

This design incorporates the bearing housing and provision for centering the probe in the gap into one component, and the same polyimide material, Vespel ${ }^{\circledR}$, used to form the probe, also functions as the bearing material (Fig. 3). One factor that allowed selection of this solution is the relatively low rotational speed (less than $1 \mathrm{~Hz}$ ) of this system during operation as well as the relatively short time the probe needed to be in operation.

Centering of the probe vertically as well as horizontal position is established by pressing the precision surfaces of the probe support against reference features on the magnet laminations. Engagement of these surfaces is accomplished by spring loaded support rods between the support assembly and a rigid beam attached to the magnet assembly.

\section{FIELD ANALYSIS}

All results are expressed according to the convention that the field is represented in terms of harmonics coefficients 


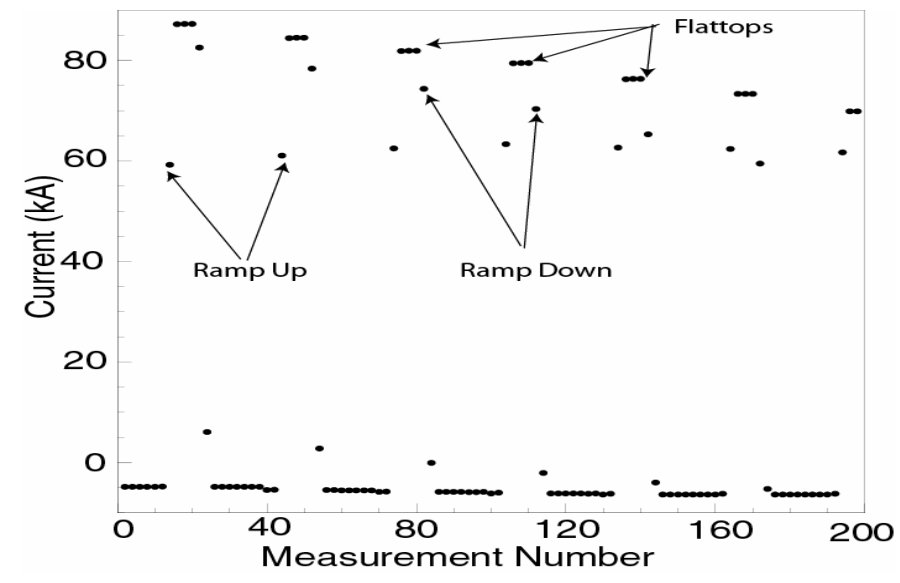

Fig. 4. Current profile during the test. The picture shows the fast ramps to flattop and back to zero current.

defined in a series expansion given by

$$
B_{y}+i B_{x}=B_{1} 10-4 \sum_{n=1}^{\infty}\left(b_{n}+i a_{n}\right)\left(\frac{x+i y}{r_{o}}\right)^{n-1}
$$

where $B_{x}$ and $B_{y}$ are the field components in the Cartesian coordinates, $b_{n}$ and $a_{n}$ are the 2n-pole normal and skew coefficients $\left(b_{1}=10^{4}\right)$, reported in units, at a reference radius $r_{0}$ of $10 \mathrm{~mm}$, approximately $2 / 3$ of the available aperture.

One of the difficulties faced in measuring a combined function magnet is to "center" the probe in the magnet. We relied on accurate mechanical measurements to determine the exact probe position inside the gap. With the rotating probe and the probe supports positioned between the poles on one side of the magnet in the space reserved for the beam pipe (Fig. 3), the mechanical measurements were performed using the combination of a depth micrometer and feeler gauges. Referencing from the lamination, the depth micrometer was used to measure the distance to the outer edge of the probe form. With this information and the previously measured probe outside diameter, the determination of the center line of the probe with respect to the lamination edge was made. This provided the horizontal position of the probe center. Next, measurements were made on either side of the probe supports with feeler gages to determine the gap between the top and bottom of the probe supports and the edge of the lamination. With this information, and the dimensions of probe support and probe, the determination of the center of the probe with

TABLE I

FIELD HARMONICS (IN UNITS AT 10 MM) AT 3.2 KA INJECTION CURRENT (LEFT) AND AT 87.5 KA COLLISION CURRENT (RIGHT)

\begin{tabular}{ccccc}
\hline \hline \multirow{2}{*}{$\begin{array}{c}\text { Harmonic } \\
\text { order }\end{array}$} & \multicolumn{2}{c}{ Injection $(0.1 \mathrm{~T})$} & \multicolumn{2}{c}{ Collision $(1.966 \mathrm{~T})$} \\
\cline { 2 - 5 } & $\mathrm{b}_{\mathrm{n}}{ }^{\text {inj }}$ & $\mathrm{a}_{\mathrm{n}}{ }^{\text {inj }}$ & $\mathrm{b}_{\mathrm{n}}{ }^{\text {col }}$ & $\mathrm{a}_{\mathrm{n}}{ }^{\text {col }}$ \\
1 & 10000 & 0. & 10000. & 0. \\
2 & -412.7 & -0.1 & -413.6 & -2.4 \\
3 & 2.8 & 2.6 & 2.56 & -0.7 \\
4 & -3.6 & -0.6 & 0.4 & 1.6 \\
5 & 2.5 & -0.8 & 6.3 & -0.3 \\
6 & 2.6 & 2.4 & 3.4 & -0.4 \\
7 & $\mathrm{n} / \mathrm{a}$ & $\mathrm{n} / \mathrm{a}$ & 1.7 & -0.8 \\
8 & $\mathrm{n} / \mathrm{a}$ & $\mathrm{n} / \mathrm{a}$ & 1.6 & 0.4 \\
9 & $\mathrm{n} / \mathrm{a}$ & $\mathrm{n} / \mathrm{a}$ & 1.9 & 1.5 \\
10 & $\mathrm{n} / \mathrm{a}$ & $\mathrm{n} / \mathrm{a}$ & -0.3 & 1.3 \\
\hline \hline
\end{tabular}

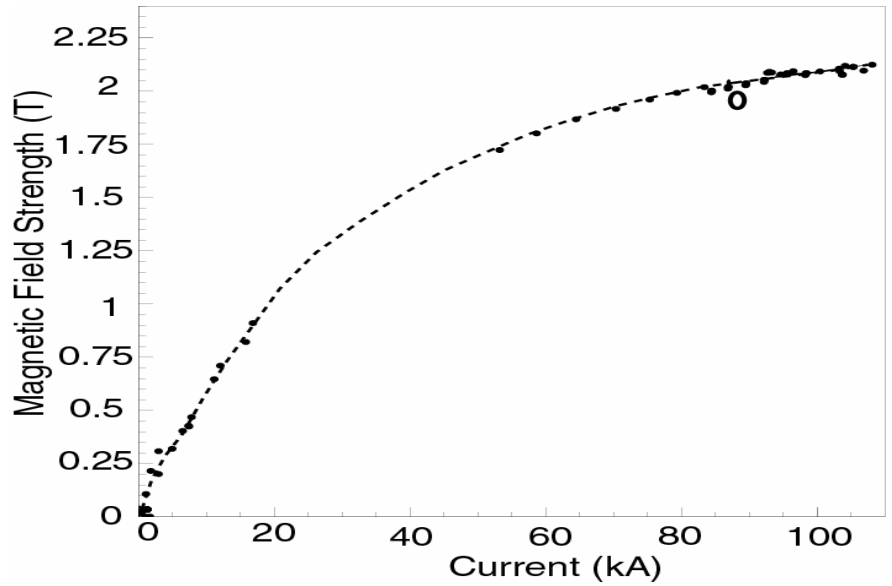

Fig. 5. The dipole strength versus the current. The open point represents the calculated dipole strength of $1.966 \mathrm{~T}$ at $87.5 \mathrm{kA}$.

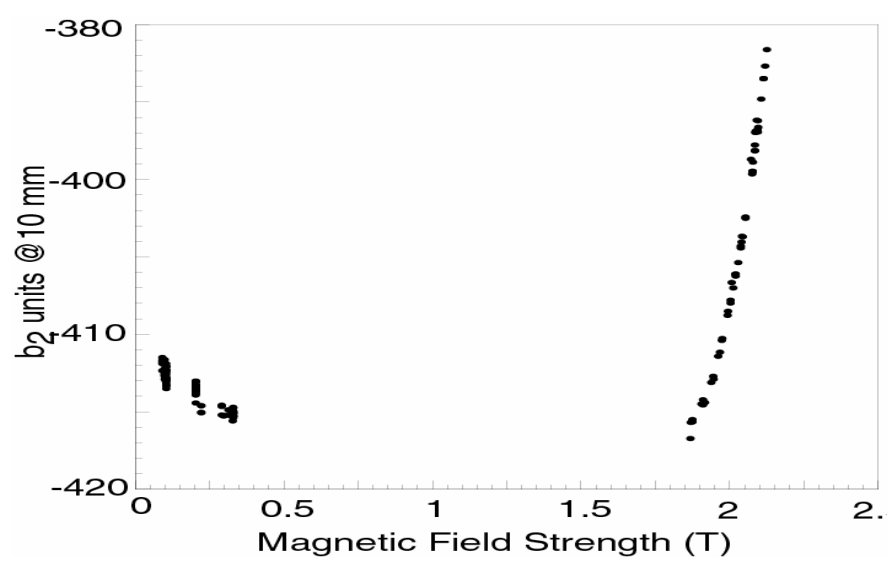

Fig. 6. The normal quadrupole component versus the main dipole field. The points on the left correspond to the measurements at injection field.

respect to the magnet gap in the vertical direction was made with an estimated uncertainty of $\pm 25 \mu \mathrm{m}$ in the horizontal direction and $\pm 50 \mu \mathrm{m}$ in the vertical.

Fig. 4 shows a part of the current ramp profile during the test period. Each point corresponds to two rotations of the probe with an angular speed of $1 \mathrm{~Hz}$. A typical experiment consisted of a fast ramp with a speed of $10 \mathrm{kA} / \mathrm{s}$ to a 6-10 s flat top between 70 and $105 \mathrm{kA}$ followed by a fast ramp back to zero. Each experiment is represented by one cycle in Fig. 4. The DAQ system was stopped between the experiments. (Note that there are time gaps between the experiments not shown on the plot.)

The dipole strength dependence on the current is shown in Fig. 5 up to $\sim 105 \mathrm{kA}, 20 \%$ more than the design operating current of $87.5 \mathrm{kA}$. The open point represents the predicted magnet strength of $1.966 \mathrm{~T}$ at $87.5 \mathrm{kA}$ [1]. A difference on the order of $4 \%$ is observed between the measurement and simulation. This difference is within the estimated systematic measurement error due to the probe calibration.

Table I summarizes the field harmonics up to order 10 at injection and collision fields. The injection plateau is defined as $0.1 \mathrm{~T}$ dipole field [1] corresponding to $3.2 \mathrm{kA}$. Measurements were taken over a relatively long $2 \mathrm{~min}$. plateau at this current. Low order harmonics, up to the 


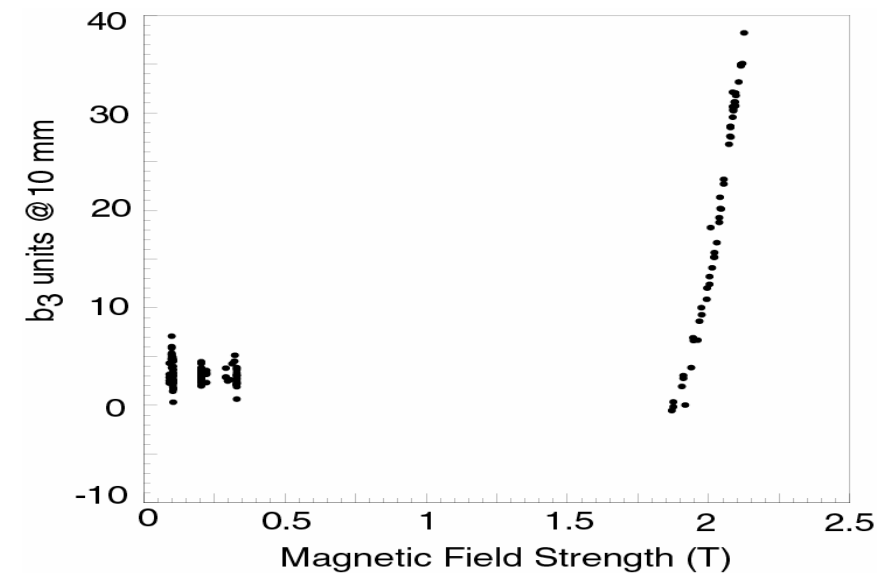

Fig. 7. The normal sextupole component versus the main dipole field. The points on the left correspond to the measurements at injection field.

dodecapole, averaged over the entire duration of the plateau are given in Table I. At this low field, we found that the probe sensitivity is insufficient for accurate determination of the higher order harmonics.

Collision harmonics were defined as the average of the all measurements with dipole field strength between 1.7 and $2.0 \mathrm{~T}$. The result is presented in the right two columns of Table I. A typical variance for the low (high) order collision harmonics is $1.4(0.8)$ units except for the normal quadrupole which has a variance of 3.1 units. One should note that most of the harmonics change with current, and their variances do not represent measurement uncertainty.

A 4\% normal quadrupole is included in the design of the combined function magnet [1]. This gradient field provides focusing of the beam and eliminates the need for separate quadrupole magnets. Our measurements (Table I and Fig. 6) show a normal quadrupole of $4.1 \%$ at injection and collision, in good agreement with that expected from the design of the magnet.

The normal sextupole as a function of the main dipole field strength is shown in Fig. 7 . One sees that $b_{3}$ increases rapidly up to $\sim 35$ units in the field region above $1.9 \mathrm{~T}$. (Note that the design operational current is $87.5 \mathrm{kA}, 1.966 \mathrm{~T}$.) A similar, although smaller, effect is observed in the normal quadrupole (Fig. 6, right). These effects are due to yoke saturation, especially in the region of the magnet poles.

\section{CONCLUSIONS}

A prototype transmission line gradient dipole magnet, proposed as the main component of a $20 \mathrm{TeV}$ Stage I VLHC, was fabricated and tested at Fermilab. Field measurements using a small, $15 \mathrm{~mm}$ diameter probe were performed. Results are in good agreement with the design parameters of the magnet: $1.966 \mathrm{~T}$ dipole field at $87.5 \mathrm{kA}, 4.1 \%$ quadrupole gradient and $\sim 3$ unit sextupole at injection and collision fields. All other normal and skew harmonics are relatively small.

\section{ACKNOWLEDGMENT}

The authors would like to acknowledge the contributions of C. Reid during the probe design, S. Helis for winding the probe and $\mathrm{C}$. Hess for technical assistance with the magnetic measurement system and for operational support.

\section{REFERENCES}

[1] G. Ambrosio et al. , "Design Study for a Staged Very Large Hadron Collider”, Fermilab-TM-2149, 2001.

[2] E. Malamud, ed., "The Pipetron: Low-Field Approach to a Very Large Hadron Collider, Snowmass '96.

[3] G. W. Foster, V. S. Kashikhin, V. V. Kashikhin, "Pole Profile Optimization of VLHC Transmission Line Magnet", in Proc. EPAC 1998, Available: http://accelconf.web.cern.ch/AccelConf/e98/PAPERS/ THP02A.PDF

[4] G. W. Foster, V. S. Kashikhin, I. Novitski, "Design of a 2 Tesla Transmition Line Magnet for VLHC", IEEE Trans. of Applied Superconductivity, Vol. 10, No. 1, March 2000, pp. 3327-3329.

[5] H. Piekarz et al., "Design, Fabrication and Test of a 2 Tesla Superconducting Transmission Line Magnet System", submitted for publication, this conference.

[6] G. W. Foster et al., "The 100 kA VLHC Transmission Line Magnet Superconducting Cable Test Facility", IEEE Trans. of Applied Superconductivity, Vol. 10, No. 1, March 2000, pp. 318-321.

[7] V. S. Kashikhin et al., "Test Results of a 2 Tesla Superconducting Transmission Line Magnet obtained with 102 Sensors Array of Hall Station", submitted for publication, this conference.

[8] PDI 5035 Precision Digital Integrator, METROLAB Instruments SA, June 1994.

[9] J.M. Nogiec, J. Sim, K. Trombly-Freytag, D. Walbridge, "EMS: A Framework for Data Acquisition and Analysis," in Proc. ACAT2000, Batavia, 2000, pp. 225-257.

[10] J.M. Nogiec et al., "A Flexible and Configurable System to Test Accelerator Magnets," in Proc. $19^{\text {th }}$ PAC, Chicago, IL, 2001, Available: http://accelconf.web.cern.ch/AccelConf/p01/PAPERS/ TPAH089.PDF

[11] G. Velev el al., "Field Quality of the LHC Inner Triplet Quadrupoles at Fermilab”, in Proc. PAC 2003, Available: http://accelconf.web.cern.ch/ AccelConf/p03/ PAPERS/WPAE015.PDF

[12] G. Velev el al., "Field Quality Measurements of the LQXB Inner Triplet Quadrupoles for LHC”, IEEE Trans. of Applied Superconductivity, Vol. 15, No. 2, June 2005, pp. 1102-1105.

[13] "Vespel ${ }^{\circledR}$ Product Guide”, E.I. du Pont Nemours and Company, Available: http://www.dupont.com/vespel/pdflit/vesprodguide.pdf. 